\title{
Adaptive Human Pilot Model for Uncertain Systems
}

\author{
S. S. Tohidi ${ }^{1}$ and Y. Yildiz ${ }^{1}$
}

\begin{abstract}
Inspired by humans' ability to adapt to changing environments, this paper proposes an adaptive human model that mimics the crossover model despite input bandwidth deviations and plant uncertainties. The proposed human pilot model structure is based on the model reference adaptive control, and the adaptive laws are obtained using the LyapunovKrasovskii stability criteria applied to the overall closed loop system including the human pilot and the plant. The proposed model can be employed for human-in-the-loop stability and performance analyses with different controllers and plant types. A numerical example is used to demonstrate the effectiveness of the presented method.
\end{abstract}

\section{INTRODUCTION}

Unique abilities of humans such as adaptive behavior in dynamic environments, and social interaction and moral judgment capabilities, make humans essential elements of many control loops, operating in close collaboration with autonomy. Compared to human control, autonomy provides higher computational performance and multi-tasking capabilities without any fatigue, stress, or boredom [1], [2].

Apart from their individual strengths, humans and autonomy have their own weaknesses. Compared to automatic control, the probability of human error causing system failure is higher. Moreover, humans may have anxiety, fear and unconsciousness during operations. In the tasks that require increased attention and focus, humans may tend to provide high gain control inputs which can cause undesired oscillations. One example of this phenomenon, for example, is the occurrence of pilot induced oscillations (PIO), where undesired and sustained oscillations are observed due to an abnormal coupling between the aircraft and the pilot [3], [4], [5], [6]. Similarly, there exists cases, where the autonomy fails due to an uncertainty, fault or cyber-attack [7]. Thus, it may be more preferable to design systems where humans and automation work in harmony, complementing each other, resulting in a structure that benefits from the advantages of both.

A mathematically rigorous investigation of human in the loop dynamics help develop safe control mechanisms, and provide a better realization and understanding of human control actions and limitations [8], [9], [10]. To achieve this purpose, reliable human mathematical models are required. One of the first human models in aeronautics is proposed by McRuer in [11] as a quasi-linear model which can be

\footnotetext{
*This effort was sponsored by the Scientific and Technological Research Council of Turkey under grant number 118E202, and by Science Academy's Young Scientist Award Program (BAGEP)

${ }^{1}$ S. S. Tohidi and Y. Yildiz are with Faculty of Mechanical Engineering, Bilkent University, Cankaya, Ankara 06800, Turkey \{shahabaldin, yyildiz\}abilkent.edu.tr
}

used in closed loop stability analysis [12]. In [13], [14] and [15], it is emphasized that every control intention has to be translated to a body movement by the neuromuscular system, and a transfer function model is proposed illustrating this observation. Crossover model, another human pilot model defined in [16], is motivated from the empirical observation that human pilots adapt their responses in such a way that the overall open loop system dynamics resembles that of a welldesigned feedback system. Several approaches are developed to identify the parameters of the two fundamental models, McRuer and neuromuscular models. In [17], a two-step method using wavelets and a windowed maximum likelihood estimation method are exploited for the estimation of timevarying pilot model parameters. In [18], the Linear Parameter Varying model identification framework is incorporated to estimate time-varying human state space representation matrices. Subsystem identification is used in [19] to model the control strategies of the human in the loop.

There also exist pilot models in the literature that mimics the adaptation ability of humans. In [20] and [21], the behavior of human in the loop is formulated and adaptive rules are provided based on expert experiences about human adaptive behavior in the control loop. The human pilot models proposed in [20] and [21] are shown, using simulations, to follow the crossover model. A survey on various human models can be found in [22] and [23].

In this paper, we built upon the earlier successful models and propose an adaptive human pilot model that modifies its behavior based on deviations in the forcing function (reference input) bandwidth and plant uncertainties. The contribution of this work is developing an adaptive human model that is shown, using rigorous mathematical analysis, to follow the crossover model, in the presence of plant uncertainties and time delays. To the best of authors' knowledge, this has not been achieved earlier in the literature. The adaptive laws are obtained based on the Lyapunov-Krasovskii stability criteria.

This paper is organized as follow. Section II presents the crossover law, and introduces the dynamics of the plant, human neuromuscular system and the reference model. $\mathrm{Ob}$ taining reference model parameters is discussed in Section III. Section IV presents the human adaptive control strategy and the stability analysis. Numerical examples are used in Section V to illustrate the effectiveness of the proposed methodology in the simulation environment. Finally, Section VI concludes the paper. 


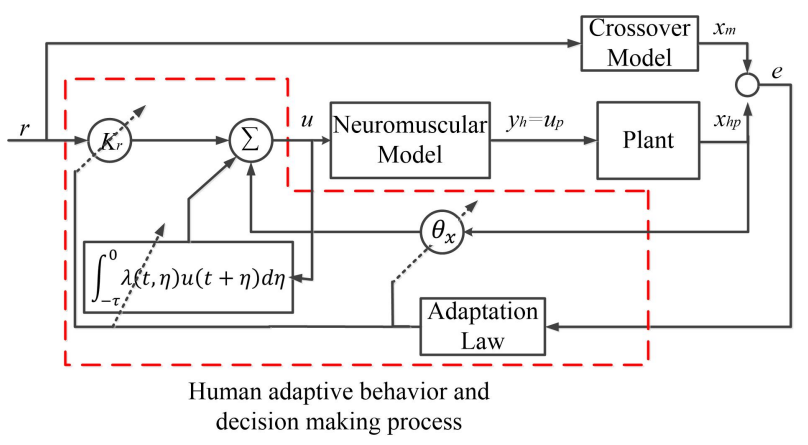

Fig. 1: The block diagram of the human adaptive behavior and decision making in closed loop system.

\section{PROBLEM STATEMENT}

According to McRuer's crossover model [16], human pilots in the control loop behave in a way that results in an open loop transfer function

$$
Y_{O L}(s)=Y_{h}(s) Y_{p}(s)=\frac{\omega_{c} e^{-\tau s}}{s},
$$

near the crossover frequency $\left(\omega_{c}\right)$, where $Y_{h}$ is the transfer function of the human pilot and $Y_{p}$ is the transfer function of the plant. $\tau$ is the effective time delay, including transport delays and high frequency neuromuscular lags.

Consider the following plant dynamics

$$
\dot{x}_{p}(t)=A_{p} x_{p}(t)+B_{p} u_{p}(t),
$$

where $x_{p} \in R^{n_{p}}$ is the plant state vector, $u_{p} \in R^{m_{p}}$ is the input vector, $A_{p} \in R^{n_{p} \times n_{p}}$ is an unknown state matrix, $B_{p} \in R^{n_{p} \times m_{p}}$ is an unknown input matrix.

The human neuromuscular model [24], [11] is represented in state space form as

$$
\begin{aligned}
\dot{x}_{h}(t) & =A_{h} x_{h}(t)+B_{h} u(t-\tau) \\
y_{h}(t) & =C_{h} x_{h}(t)+D_{h} u(t-\tau),
\end{aligned}
$$

where $x_{h} \in R^{n_{h}}$ is the neuromuscular state vector, $A_{h} \in$ $R^{n_{h} \times n_{h}}$ is the state matrix, $B_{h} \in R^{n_{h} \times m_{h}}$ is the input matrix, $C_{h} \in R^{m_{p} \times n_{h}}$ is the output matrix and $D_{h} \in$ $R^{m_{p} \times m_{h}}$ is the control output matrix. $u \in R^{m_{h}}$ is the neuromuscular input vector, which represents the control decisions taken by the human and fed to the neuromuscular system, $y_{h} \in R^{m_{p}}$ is the output vector, and $\tau \in R^{+}$is a known, constant delay. The neuromuscular model parameters are assumed to be known and the output of the model, $y_{h}$, is used as the plant input $u_{p}$ in (2), that is $y_{h}=u_{p}$ (see fig. $1)$.

By combining the human pilot and plant states, we obtain the open loop human-plant dynamics as

$$
\begin{aligned}
\underbrace{\left[\begin{array}{c}
\dot{x}_{h}(t) \\
\dot{x}_{p}(t)
\end{array}\right]}_{\dot{x}_{h p}(t)} & =\underbrace{\left[\begin{array}{cc}
A_{h} & 0_{n_{h} \times n_{p}} \\
B_{p} C_{h} & A_{p}
\end{array}\right]}_{A_{h p}} \underbrace{\left[\begin{array}{c}
x_{h}(t) \\
x_{p}(t)
\end{array}\right]}_{x_{h p(t)}} \\
& +\underbrace{\left[\begin{array}{c}
B_{h} \\
B_{p} D_{h}
\end{array}\right]}_{B_{h p}} u(t-\tau),
\end{aligned}
$$

which can be written in the following compact form

$$
\dot{x}_{h p}(t)=A_{h p} x_{h p}(t)+B_{h p} u(t-\tau),
$$

where $x_{h p}=\left[\begin{array}{ll}x_{h}^{T} & x_{p}^{T}\end{array}\right]^{T} \in R^{\left(n_{p}+n_{h}\right)}, A_{h p} \in$ $R^{\left(n_{p}+n_{h}\right) \times\left(n_{p}+n_{h}\right)}, B_{h p} \in R^{\left(n_{p}+n_{h}\right) \times m_{h}}$.

The goal is to obtain the input $u(t)$ in (3), which is the control decision of the pilot, such that the closed loop system consisting of the adaptive human pilot model and the plant follow the output of a unity feedback reference model with an open loop crossover transfer function. The closed loop transfer function of the reference model is therefore calculated as

$$
G_{c l}(s)=\frac{\frac{\omega_{c}}{s} e^{-\tau s}}{1+\frac{\omega_{c}}{s} e^{-\tau s}}=\frac{\omega_{c} e^{-\tau s}}{s+\omega_{c} e^{-\tau s}} .
$$

An approximation of (6) can be given as

$$
\hat{G}_{c l}(s)=\frac{b_{m} s^{m}+b_{m-1} s^{m-1}+\ldots+b_{0}}{s^{n}+a_{n-1} s^{n-1}+\ldots+a_{0}} e^{-\tau s},
$$

where $n=n_{h}+n_{p}, m \leq n$ are positive real constants, and $a_{i}$ and $b_{j}$ for $i=0, \ldots, n-1$ and $j=0, \ldots, m-1$, are real constants to be estimated. The reference model then can be obtained as the state space representation of (7) as

$$
\dot{x}_{m}(t)=A_{m} x_{m}(t)+B_{m} r(t-\tau),
$$

where $x_{m} \in R^{\left(n_{h}+n_{p}\right)}$ is the reference model state vector, $A_{m} \in R^{\left(n_{h}+n_{p}\right) \times\left(n_{h}+n_{p}\right)}$ is the state matrix, $B_{m} \in$ $R^{\left(n_{h}+n_{p}\right) \times m_{h}}$ is the input matrix, and $r \in R^{m_{h}}$ is the reference input.

\section{REFERENCE MODEL PARAMETERS}

The crossover transfer function (1) contains the crossover frequency, $\omega_{c}$, which is not known a priori. Experimental data, showing the reference input $(r(t))$ frequency bandwidth, $\omega_{i}$, versus crossover frequency $\omega_{c}$, is provided in [14] and [16], for plant transfer functions $K, K / s$ and $K / s^{2}$. We fit polynomials to these experimental results to obtain the crossover frequency of the open loop transfer function given a reference input frequency bandwidth. These polynomials are given in Table I. It is noted that when the reference input has multiple frequency components, the highest frequency is used to calculate the crossover frequency.

Remark 1. In this work, we use the polynomial relationships provided in Table I for zero, first and second order plant dynamics with nonzero poles and zeros. Further experimental work with humans are planned by the authors to obtain more precise crossover vs reference input frequency relationships. 


\section{TABLE I}

\begin{tabular}{|c|c|}
\hline $\begin{array}{c}\text { Plant transfer } \\
\text { function }\end{array}$ & $\begin{array}{c}\text { Crossover frequency of the } \\
\text { open loop transfer function }\end{array}$ \\
\hline$K$ & $\omega_{c}=0.067 \omega_{i}^{2}+0.099 \omega_{i}+4.8$ \\
\hline$K / s$ & $\omega_{c}=0.14 \omega_{i}+4.3$ \\
\hline$K / s^{2}$ & $\omega_{c}=-0.0031 \omega_{i}^{4}-0.072 \omega_{i}^{3}+0.29 \omega_{i}^{2}$ \\
& $-0.13 \omega_{i}+3$ \\
\hline
\end{tabular}

\section{HUMAN CONTROL COMMAND}

The adaptive human decision command, $u(t)$, is determined as

$$
u(t)=K_{r} K_{x} x_{h p}(t+\tau)+K_{r} r(t)
$$

where $K_{x} \in R^{m_{h} \times\left(n_{h}+n_{p}\right)}$, and $K_{r} \in R^{m_{h} \times m_{h}}$. Using (9) and (5), the closed loop dynamics can be obtained as

$$
\dot{x}_{h p}(t)=\left(A_{h p}+B_{h p} K_{r} K_{x}\right) x_{h p}(t)+B_{h p} K_{r} r(t-\tau) .
$$

Equation (9) describes a non-causal decision command which requires future values of the states. This problem can be eliminated by solving the differential equation (5) as a $\tau$-seconds ahead predictor as

$$
x_{h p}(t+\tau)=e^{A_{h p} \tau} x_{h p}(t)+\int_{-\tau}^{0} e^{-A_{h p} \eta} B_{h p} u(t+\eta) d \eta .
$$

Assumption 1. There exist ideal parameters $K_{r}^{*}$ and $K_{x}^{*}$ satisfying the following matching conditions

$$
\begin{aligned}
& A_{h p}+B_{h p} K_{r}^{*} K_{x}^{*}=A_{m} \\
& B_{h p} K_{r}^{*}=B_{m} .
\end{aligned}
$$

By substituting (11) into (9), the control input can be written as

$$
\begin{aligned}
u(t) & =K_{r} K_{x} e^{A_{h p} \tau} x_{h p}(t) \\
& +K_{r} K_{x} \int_{-\tau}^{0} e^{-A_{h p} \eta} B_{h p} u(t+\eta) d \eta+K_{r} r(t) .
\end{aligned}
$$

By defining $\theta_{x}(t)$ and $\lambda(t, \eta)$ as

$$
\begin{aligned}
\theta_{x}(t) & =K_{r}(t) K_{x}(t) e^{A_{h p} \tau}, \\
\lambda(t, \eta) & =K_{r}(t) K_{x}(t) e^{-A_{h p} \eta} B_{h p},
\end{aligned}
$$

the controller (13) can be written as (see fig. 1)

$$
u(t)=\theta_{x}(t) x_{h p}(t)+\int_{-\tau}^{0} \lambda(t, \eta) u(t+\eta) d \eta+K_{r}(t) r(t) .
$$

The ideal values of $\theta_{x}$ and $\lambda$ can be obtained as

$$
\begin{aligned}
\theta_{x}^{*} & =K_{r}^{*} K_{x}^{*} e^{A_{h p} \tau} \\
\lambda^{*}(\eta) & =K_{r}^{*} K_{x}^{*} e^{-A_{h p} \eta} B_{h p} .
\end{aligned}
$$

Since $A_{h p}$ and $B_{h p}$ are unknown, $\theta_{x}$ and $\lambda$ need to be estimated. The closed loop dynamics can be obtained using (5) and (15) as

$$
\begin{aligned}
\dot{x}_{h p}(t) & =A_{h p} x_{h p}(t)+B_{h p} \theta_{x}(t-\tau) x_{h p}(t-\tau) \\
& +\int_{-\tau}^{0} B_{h p} \lambda(t-\tau, \eta) u(t+\eta-\tau) d \eta \\
& +B_{h p} K_{r} r(t-\tau),
\end{aligned}
$$

Defining the deviations of the adaptive parameters from their ideal values as $\tilde{\theta}_{x}=\theta_{x}-\theta_{x}^{*}$ and $\tilde{\lambda}=\lambda-\lambda^{*}$, and adding and subtracting $A_{m} x_{h p}(t)$ to (17), and using (12), we have

$$
\begin{aligned}
\dot{x}_{h p}(t) & =A_{m} x_{h p}(t)-B_{h p} K_{r}^{*} K_{x}^{*} x_{h p}(t) \\
& +B_{h p} K_{r}(t-\tau) K_{x}(t-\tau)\left(e^{A_{h p} \tau} x_{h p}(t-\tau)\right. \\
& \left.+\int_{-\tau}^{0} e^{-A_{h p} \eta} B_{h p} u(t+\eta-\tau) d \eta\right) \\
& +B_{h p} K_{r}(t-\tau) r(t-\tau) .
\end{aligned}
$$

Using (11), (18) is rewritten as

$$
\begin{aligned}
\dot{x}_{h p}(t) & =A_{m} x_{h p}(t)-B_{h p} K_{r}^{*} K_{x}^{*} x_{h p}(t) \\
& +B_{h p} K_{r}(t-\tau) K_{x}(t-\tau) x_{h p}(t) \\
& +B_{h p} K_{r}(t-\tau) r(t-\tau) .
\end{aligned}
$$

Defining the tracking error as $e(t)=x_{h p}-x_{m}$, and subtracting (8) from (19), and using (12), and following the same procedure as given in [25] for unknown input matrices, we have

$$
\begin{aligned}
\dot{e}(t) & =\dot{x}_{h p}-\dot{x}_{m} \\
& =A_{m} e(t)+B_{m}\left(\tilde{K}_{x}(t-\tau) x_{h p}(t)\right. \\
& +B_{m}\left(K_{r}^{*-1}-K_{r}^{-1}(t-\tau)\right) K_{r}(t-\tau) K_{x}(t-\tau) x_{h p}(t) \\
& +B_{m}\left(K_{r}^{*-1}-K_{r}^{-1}(t-\tau)\right) K_{r}(t-\tau) r(t-\tau) .
\end{aligned}
$$

Using (11) and defining $\Phi=K_{r}^{*-1}-K_{r}^{-1}$, we can rewrite (20) as

$$
\begin{aligned}
\dot{e}(t) & =A_{m} e(t)+B_{m} K_{r}^{*-1}\left(K_{r}^{*} K_{x}(t-\tau)-K_{r}^{*} K_{x}^{*}\right) \\
& \times\left(e^{A_{h p} \tau} x_{h p}(t-\tau)+\int_{-\tau}^{0} e^{-A_{h p} \eta} B_{h p} u(t+\eta-\tau) d \eta\right) \\
& +B_{m} \Phi(t-\tau)\left(K _ { r } ( t - \tau ) K _ { x } ( t - \tau ) \left(e^{A_{h p} \tau} x_{h p}(t-\tau)\right.\right. \\
& \left.+\int_{-\tau}^{0} e^{-A_{h p} \eta} B_{h p} u(t+\eta-\tau) d \eta\right) \\
& \left.+K_{r}(t-\tau) r(t-\tau)\right) .
\end{aligned}
$$

Using (16) and (21), we obtain that

$$
\begin{aligned}
\dot{e}(t) & =A_{m} e(t)+B_{m} K_{x}(t-\tau)\left(e^{A_{h p} \tau} x_{h p}(t-\tau)\right. \\
& \left.+\int_{-\tau}^{0} e^{-A_{h p} \eta} B_{h p} u(t+\eta-\tau) d \eta\right) \\
& -B_{m} K_{r}^{*-1}\left(\theta_{x}^{*} x_{h p}(t-\tau)\right. \\
& \left.+\int_{-\tau}^{0} \lambda^{*}(\eta) u(t+\eta-\tau) d \eta\right) \\
& +B_{m} \Phi(t-\tau) u(t-\tau)
\end{aligned}
$$


Using (14) and (22), we obtain that

$$
\begin{aligned}
\dot{e}(t) & =A_{m} e(t)+B_{m}\left(\left(K_{r}^{-1}(t-\tau) \theta_{x}(t-\tau)-K_{r}^{*-1} \theta_{x}^{*}\right)\right. \\
& \times x_{h p}(t-\tau)+\int_{-\tau}^{0}\left(K_{r}^{-1}(t-\tau) \lambda(t-\tau, \eta)\right. \\
& \left.\left.-K_{r}^{*-1} \lambda^{*}(\eta)\right) u(t+\eta-\tau) d \eta\right) \\
& +B_{m} \Phi(t-\tau) u(t-\tau) .
\end{aligned}
$$

Defining $\theta_{1}=K_{r}^{-1} \theta_{x}$ and $\lambda_{1}=K_{r}^{-1} \lambda$, and using their deviations from their ideal values $\tilde{\theta}_{1}=\theta_{1}-\theta_{1}^{*}$ and $\tilde{\lambda}_{1}=$ $\lambda_{1}-\lambda_{1}^{*}$, where $\theta_{1}^{*}=K_{r}^{*-1} \theta_{x}^{*}$ and $\lambda_{1}^{*}=K_{r}^{*-1} \lambda^{*}$, we can rewrite (23) as

$$
\begin{aligned}
\dot{e}(t) & =A_{m} e(t)+B_{m} \tilde{\theta}_{1}(t-\tau) x_{h p}(t-\tau) \\
& +B_{m} \int_{-\tau}^{0} \tilde{\lambda}_{1}(t-\tau, \eta) u(t+\eta-\tau) d \eta \\
& +B_{m} \Phi(t-\tau) u(t-\tau)
\end{aligned}
$$

Theorem 1. Given the initial conditions $\tilde{\theta}_{1}(\xi), \tilde{\lambda}_{1}(\xi, \eta), \Phi(\xi)$ and $x_{h p}(\xi)$ for $\xi \in[-\tau, 0]$, and $u(\zeta)$ for $\zeta \in[-2 \tau, 0]$, there exists a $\tau^{*}$ such that for all $\tau \in\left[0, \tau^{*}\right]$, the humanplant system (5), with the controller (15), and the following adaptive laws

$$
\begin{gathered}
\dot{\theta}_{1}^{T}(t)=-x_{h p}(t-\tau) e(t)^{T} P B_{m}, \\
\dot{\Phi}^{T}(t)=-u(t-\tau) e(t)^{T} P B_{m}, \\
\dot{\lambda}_{1}^{T}(t, \eta)=-u(t+\eta-\tau) e(t)^{T} P B_{m},
\end{gathered}
$$

where $P$ is the symmetric positive definite matrix satisfying the Lyapunov equation $A_{m}^{T} P+P A_{m}=-Q$ for a symmetric positive definite matrix $Q$, follow the crossover model (8), while all the signals remain bounded. It is noted that the controller parameters can be obtained using $\dot{K}_{r}=K_{r} \dot{\Phi} K_{r}$, $\theta_{x}(t)=K_{r}(t) \theta_{1}(t)$ and $\lambda(t)=K_{r}(t) \lambda_{1}(t)$.

Proof. Consider a Lyapunov-Krasovskii functional ([26]; [27])

$$
\begin{aligned}
V(t) & =e^{T} \operatorname{Pe}+\operatorname{tr}\left(\Phi^{T}(t) \Phi(t)\right)+\operatorname{tr}\left(\tilde{\theta}_{1}^{T}(t) \tilde{\theta}_{1}(t)\right) \\
& +\int_{-\tau}^{0} \int_{t+v}^{t} \operatorname{tr}\left(\dot{\tilde{\theta}}_{1}^{T}(\xi) \dot{\tilde{\theta}}_{1}(\xi)\right) d \xi d v \\
& +\int_{-\tau}^{0} \int_{t+v}^{t} \operatorname{tr}\left(\dot{\Phi}^{T}(\xi) \dot{\Phi}(\xi)\right) d \xi d v \\
& +\int_{-\tau}^{0} \operatorname{tr}\left(\tilde{\lambda}_{1}^{T}(t, \eta) \tilde{\lambda}_{1}(t, \eta)\right) d \eta \\
& +\int_{-\tau}^{0} \int_{t+v}^{t} \int_{-\tau}^{0} \operatorname{tr}\left(\dot{\tilde{\lambda}}_{1}^{T}(\xi, \eta) \dot{\tilde{\lambda}}_{1}(\xi, \eta)\right) d \eta d \xi d v
\end{aligned}
$$

where $\dot{\tilde{\lambda}}_{1}=\frac{\partial \tilde{\lambda}_{1}}{\partial t}$. The derivative of $V(t)$ can be calculated by using Leibniz's rule, that is $\frac{d}{d t} \int_{a(t)}^{b(t)} f(y) d y=f(b(t)) \frac{d b(t)}{d t}-$ $f(a(t)) \frac{d a(t)}{d t}$, and the trace operator property $\operatorname{tr}\left(X^{T} X\right)=$
$\|X\|_{F}^{2}$, as

$$
\begin{aligned}
\dot{V}(t) & =\dot{e}^{T}(t)^{T} P e(t)+e^{T}(t) P \dot{e}(t)+2 \operatorname{tr}\left(\dot{\tilde{\theta}}_{1}^{T}(t) \tilde{\theta}_{1}(t)\right) \\
& +2 \operatorname{tr}\left(\dot{\Phi}^{T}(t) \Phi(t)\right)+\int_{-\tau}^{0} 2 \operatorname{tr}\left(\dot{\tilde{\lambda}}_{1}^{T}(t, \eta) \tilde{\lambda}_{1}(t, \eta)\right) d \eta \\
& +\tau\left\|\dot{\tilde{\theta}}_{1}(t)\right\|_{F}^{2}-\int_{-\tau}^{0}\left\|\dot{\tilde{\theta}}_{1}(t+v)\right\|_{F}^{2} d v \\
& +\tau\|\dot{\Phi}(t)\|_{F}^{2}-\int_{-\tau}^{0}\|\dot{\Phi}(t+v)\|_{F}^{2} d v \\
& +\tau \int_{-\tau}^{0}\left\|\dot{\tilde{\lambda}}_{1}(t, \eta)\right\|_{F}^{2} d \eta \\
& -\int_{-\tau}^{0} \int_{-\tau}^{0}\left\|\dot{\tilde{\lambda}}_{1}(t+v, \eta)\right\|_{F}^{2} d \eta d v .
\end{aligned}
$$

Using (24)-(27), the upper bound of (29) can be obtained as

$$
\begin{aligned}
\dot{V}(t) & \leq-e^{T}(t) Q e(t) \\
& +2 \tau \operatorname{tr}\left(e(t) x_{h p}^{T}(t-\tau) x_{h p}(t-\tau) e(t)^{T}\right) \operatorname{tr}\left(P B_{m} B_{m}^{T} P\right) \\
& +2 \tau \operatorname{tr}\left(e(t) u^{T}(t-\tau) u(t-\tau) e(t)^{T}\right) \operatorname{tr}\left(P B_{m} B_{m}^{T} P\right) \\
& +2 \tau \int_{-\tau}^{0} \operatorname{tr}\left(e(t) u^{T}(t-\tau+\eta) u(t-\tau+\eta) e(t)^{T}\right) \\
& \times \operatorname{tr}\left(P B_{m} B_{m}^{T} P\right) d \eta \\
& \leq-\lambda_{\min }(Q)\|e(t)\|^{2} \\
& +2 \tau\left\|x_{h p}(t-\tau) e(t)^{T}\right\|_{F}^{2}\left\|B_{m}^{T} P\right\|_{F}^{2} \\
& +2 \tau\left\|u(t-\tau) e(t)^{T}\right\|_{F}^{2}\left\|B_{m}^{T} P\right\|_{F}^{2} \\
& +2 \tau \int_{-\tau}^{0}\left\|u(t+\eta-\tau) e(t)^{T}\right\|_{F}^{2}\left\|B_{m}^{T} P\right\|_{F}^{2} d \eta \\
& \leq-\lambda_{\min }(Q)\|e(t)\|^{2} \\
& +2 \tau\left\|x_{h p}(t-\tau)\right\|^{2}\|e(t)\|^{2}\left\|B_{m}^{T} P\right\|_{F}^{2} \\
& +2 \tau\|u(t-\tau)\|^{2}\|e(t)\|^{2}\left\|B_{m}^{T} P\right\|_{F}^{2} \\
& +2 \tau \int_{-\tau}^{0}\|u(t+\eta-\tau)\|^{2}\|e(t)\|^{2}\left\|_{m}^{T} P\right\|_{F}^{2} d \eta \\
& =\left\|B_{m}^{T} P\right\|\left\|_{F}^{2}\right\| e(t) \|^{2}\left(-\frac{\lambda_{m i n}(Q)}{\left\|B_{m}^{T} P\right\|_{F}^{2}}\right. \\
& +2 \tau\left(\left\|x_{h p}(t-\tau)\right\|^{2}+\|u(t-\tau)\|^{2}\right. \\
& \left.\left.+\int_{-\tau}^{0}\|u(t+\eta-\tau)\|^{2} d \eta\right)\right) .
\end{aligned}
$$

Defining $q \equiv \frac{\lambda_{\min }(Q)}{\left\|B_{m}^{T} P\right\|_{F}^{2}}$, for the non-positiveness of $\dot{V}(t)$, we need to satisfy

$$
\begin{gathered}
q-2 \tau\left(\left\|x_{h p}(t-\tau)\right\|^{2}+\|u(t-\tau)\|^{2}+\right. \\
\left.+\int_{-\tau}^{0}\|u(t+\eta-\tau)\|^{2} d \eta\right) \geq 0
\end{gathered}
$$

It can be shown using proof by induction that (31) is satisfied for all $t>t_{0}$ and $\tau \in\left[0, \tau^{*}\right]$, and all the signals of the system are bounded. Then, using Barbalat's Lemma, it can be shown that the error between the human-in-theloop system output $x_{h p}$ and the reference model output $x_{m}$ converges to zero. It is noted that the error dynamics (24), the adaptive laws (25)-(27), the Lyapunov function candidate 


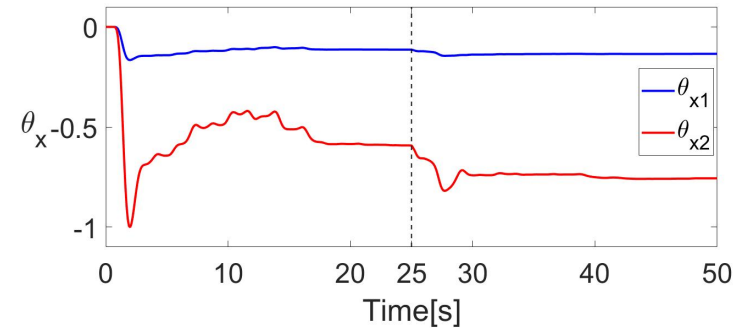

Fig. 2: Evolution of human adaptive parameters $\theta_{x_{1}}$ and $\theta_{x_{2}}$.

(28) and the inequality (31) that needs to be satisfied to show the non-positiveness of the Lyapunov function candidate are similar to those given in [26]. Therefore, the procedure that needs to be followed to complete the proof can be found in [26] and omitted here.

The above analysis implies the global stability in $\left\{e, \tilde{\theta}_{1}, \Phi, \tilde{\lambda}_{1}\right\}$ space. However, we are interested in $\left\{e, \tilde{\theta}_{1}, \tilde{K}_{r}, \tilde{\lambda}_{1}\right\}$ space since $K_{r}$, not $\Phi$, is used in the calculation of the control signal. Since $\Phi=K_{r}^{*-1}-K_{r}^{-1}$, to ensure the boundedness of all signals in the closed loop system, projection algorithm [30] can be used in the adaptive law for $K_{r}$ as:

$$
\dot{K}_{r}=\operatorname{Proj}\left(K_{r},-K_{r} B_{m}^{T} P e(t) u^{T}(t-\tau) K_{r}\right) .
$$

Remark 2. In order to implement the control signal (15), the integral term is approximated as

$$
\begin{aligned}
& \int_{-\tau}^{0} \lambda(t, \eta) u(t+\eta) d \eta \\
& \approx\left(\lambda_{1}(t) u(t-\Delta t)+\ldots+\lambda_{m}(t) u(t-m \Delta t)\right) \Delta t .
\end{aligned}
$$

\section{Simulation Results}

A first order plant $Y_{p}(s)=\frac{4}{s+1}$ is considered. The neuromuscular dynamics of the human is given as $Y_{h}(s)=$ $\frac{s+3}{s+2} e^{-0.3 s}$, where the time delay $\tau=0.3$ is the effective time delay, including human decision making delay and neuromuscular lags. The reference signal $r(t)$ is generated as a sum of the sinusoid functions with frequencies of $0.16,0.4,0.86,1.33$ and $4.2 \mathrm{rad} / \mathrm{s}$ with the same amplitude of 0.2. Thus, the highest frequency component, which is used in crossover frequency calculations, is $\omega_{i}=4.2 \mathrm{rad} / \mathrm{s}$. Employing Table I for the first order plant $Y_{p}$, the crossover frequency is calculated as $\omega_{c}=4.88 \mathrm{rad} / \mathrm{s}$. Furthermore, the reference model can be determined as the state space representation of $\hat{G}_{c l}(s)=\frac{3.8 s+24.25}{s^{2}+0.68 s+24.7} e^{-0.3 s}$, which is obtained by approximating $G_{c l}(s)=\frac{4.88 e^{-0.3 s}}{s+4.88 e^{-0.3 s}}$ using MATLAB system identification toolbox.

The overall system, whose block diagram is given in figure 1 , is simulated using the mentioned reference signal and introducing an anomaly at $t=25 \mathrm{~s}$, which is modeled by changing the plant model to $Y_{p}(s)=\frac{2}{s+0.5}$. Figures 2-4 illustrate the time evolution of human adaptive parameters, the adaptation laws that are used to obtain which are provided in (25)-(27). It is noted that a four-point discretization is

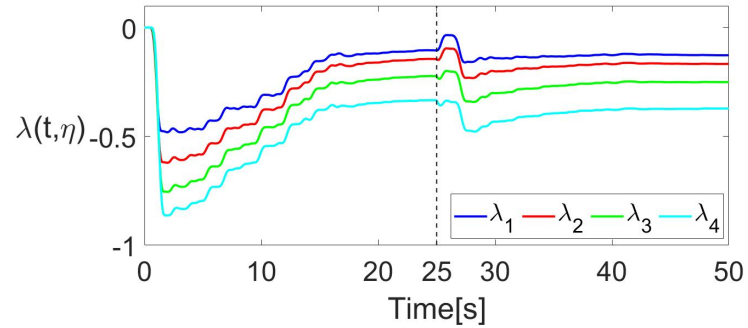

Fig. 3: Evolution of human adaptive parameters $\lambda_{i}, i=$ $1,2,3$ and 4 .

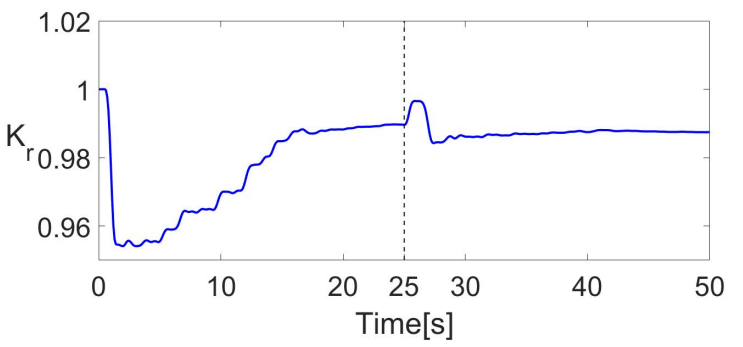

Fig. 4: Evolution of human adaptive parameter $K_{r}$.

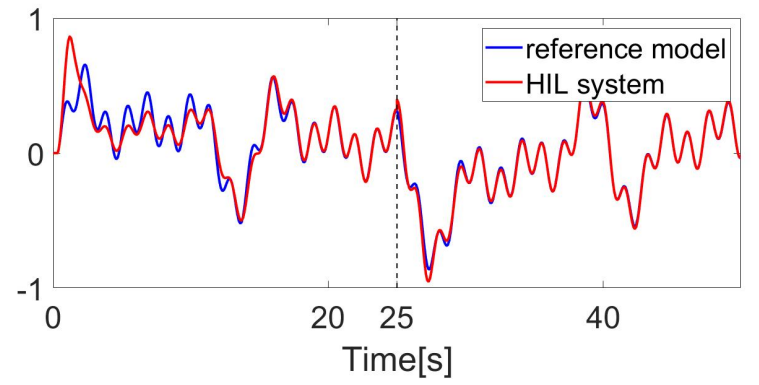

Fig. 5: Time evolution of the human-in-the-loop (HIL) system output $x_{h p}$ and the reference model output $x_{m}$.

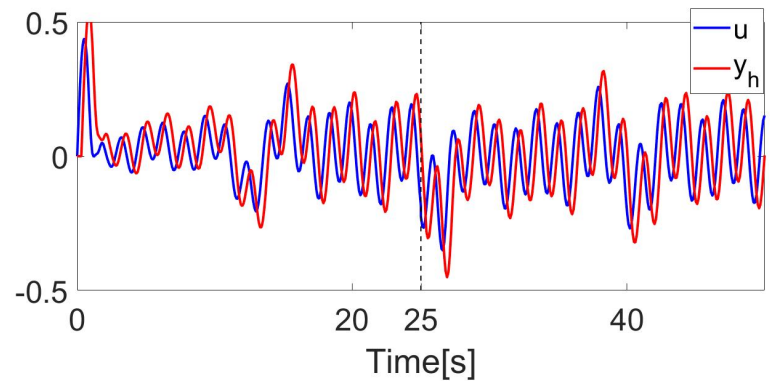

Fig. 6: Human adaptive decision-making signal $u$ and the human output $\left(y_{h}\right)$. 
used to approximate the integral in (15). Figure 5 shows how the human-plant system output $x_{h p}$ is able to follow the crossover reference model output $x_{m}$, before and after the anomaly at $t=25 \mathrm{~s}$. In figure 6 , the human decision-making signal $u(t)$ is depicted together with the neuromuscular system output $y_{h}$. It is seen that the neuromuscular dynamics slightly amplifies and delays the decision signal.

\section{CONCLUSIONS}

In this paper, an adaptive human pilot model with time delay, operating based on model reference adaptive control principles, is proposed. This model mimics the pilot decision making process by making sure that the overall closed loop system follows the crossover model in the presence of plant uncertainties. The stability of the system is shown using the Lyapunov-Krasovskii stability criteria. It is shown via simulations that the proposed pilot model is able to track the crossover model even after an anomaly is introduced to the system.

\section{ACKNOWLEDGMENT}

This effort was sponsored by the Scientific and Technological Research Council of Turkey under grant number 118E202, and by Science Academy's Young Scientist Award Program (BAGEP).

\section{REFERENCES}

[1] W. D. Nothwang, R. M. Robinson, S. A. Burdenand, M. J. McCourt, and J. W. Curtis, "The human should be part of the control loop?," Resilience Week (RWS), 2016.

[2] M. Körber, W. Schneider, and M. Zimmermann, "Vigilance, boredom proneness and detection time of a malfunction in partially automated driving," International Conference on Collaboration Technologies and Systems (CTS), 2015.

[3] D. M. Acosta, Y. Yildiz, R. W. Craun, S. D. Beard, M. W. Leonard, G. H. Hardy, and M. Weinstein, "Piloted evaluation of a control allocation technique to recover from pilot-induced oscillations," J. Aircraft, vol. 52, pp. 130-140, 2015.

[4] Y. Yildiz, and I. V. Kolmanovsky, "A control allocation technique to recover from pilot-induced oscillations (CAPIO) due to actuator rate limiting," American Control Conference (ACC), pp. 516-523, 2010.

[5] Y. Yildiz, I. V. Kolmanovsky, and D. Acosta, "A control allocation system for automatic detection and compensation of phase shift due to actuator rate limiting," American Control Conference (ACC), pp. 444-449, 2011.

[6] S. S. Tohidi, Y. Yildiz, and I. Kolmanovsky, "Pilot induced oscillation mitigation for unmanned aircraft systems: an adaptive control allocation approach," IEEE Conference on Control Technology and Applications (CCTA), Copenhagen, Denmark, Aug. 2018.

[7] W. Li, D. Sadigh, S. S. Sastry, and S. A. Seshia, "Synthesis for humanin-the-loop control systems," TACAS Conference, 2014.

[8] T. Yucelen, Y. Yildiz, R. Sipahi, E. Yousefi, and N. Nguyen, "Stability limit of human-in-the-loop model reference adaptive control architecture," International Journal of Control, vol. 91, pp. 2314-2331, 2018.

[9] M. Xia, A. Rahnama, S. Wang, and P. J. Antsaklis, "On guaranteeing passivity and performance with a human controller," Mediterranean Conference on Control and Automation, Torremolinos, Spain, Jul. 2015.

[10] R. Sipahi, and, S. -I. Niculescu, "Stability of car following with human memory effects and automatic headway compensation," Phil. Trans. R. Soc., vol. 368, pp. 4563-4583, 2010.

[11] D. McRuer, and E. Krendel, "Mathematical models of human pilot behaviour," AGAR-Dograph AGARD-AG-188, Advisory Group for Aerospace Research \& Development, 1974.

[12] T. P. Neal, and R. E. Smith, "An in-flight investigation to develop control system design criteria for fighter airplanes," Tech.Rep.AFFDLTR-70-74, Wright-Patterson Air Force Base, Ohio, USA: Air Force Flight Dynamics Laboratory, 1970.
[13] D. T. McRuer, and E. S. Krendel, "Human pilot dynamics in compensatory systems: theory, models, and experiments with controlled element and forcing function variations," Tech.Rep.AFFDL-TR-6515, Wright-Patterson Air Force Base, Ohio, USA: Air Force Flight Dynamics Laboratory, 1965.

[14] G. C. Beerens, H. J. Damveld, M. Mudler, and M. M. van Paassen, "An investigation into crossover regression and pilot parameter adjustment," AIAA Modeling and Simulation Technologies Conference and Exhibit, 2008.

[15] M. Mudler, D. M. Pool, D. A. Abbink, E. R. Boer, P. M. T. Zaal, F. M. Drop, K. van der El, and M. M. van Paassen, "Manual control cybernetics: state-of-the-art and current trends," IEEE Transactions on Human-Machine Systems, vol. 48, 2018.

[16] D. T. Mcruer, and H. Jex, "A review of quasi-linear pilot models," IEEE Transactions on human Factors in Electronics, vol. 8, pp. 231249, 1967.

[17] P. M. T. Zaal, and B. T. Sweet, "Estimation of time-varying pilot model parameters," AIAA Modeling and Simulation Technologies Conference, Portland, OR, USA, Aug. 2011.

[18] R. F. M. Duarte, D. M. Pool, and M. M. van Paassen, "Experimental scheduling functions for global LPV Human controller modeling," IFAC, Jul. 2017.

[19] X. Zhang,T. M . Seigler, and J. B. Hoagg, "Modeling the control strategies that humans use to control nonminimum-phase systems," American Control Conference, Chicago, IL, USA, Jul. 2015.

[20] R. A. Hess, "Modeling pilot control behavior with sudden changes in vehicle dynamics," Journal of Aircraft, vol. 46, pp. 1584-1592, 2009.

[21] R. A. Hess, "Modeling human pilot adaptation to flight control anomalies and changing task demands," Journal of Guidance, Control and dynamics, vol. 39, pp. 655-666, 2016.

[22] M. Lone, and A. Cooke, "Review of pilot models used in aircraft flight dynamics," Aerospace Science and Technology, vol. 34, pp. 55$74,2014$.

[23] S. Xu, W. Tan, A. V. Efremov, L. Sun, and X. Qu, "Review of control models for human pilot behavior," Annual Reviews in Control, vol. 44, pp. 274-291, 2017.

[24] R. E. Magdaleno, and X. Qu, "Experimental validation and analytical elaboration for models of the pilot's neuromuscular subsystem in tracking tasks," Systems Technology, Inc., Hawthorne, California, 1971.

[25] K. S. Narendra, and A. M. Annaswamy, "Stable adaptive systems," Dover Publications, New York, USA, 2004.

[26] Y. Yildiz, A. Annaswamy, I. Kolmanovsky, and D. Yanakiev, "Adaptive posicast controller for time-delay systems with relative degree $n^{*} \leq$ 2,"Automatica, vol. 46, pp. 279-289, 2010.

[27] S. -I. Niculescu, and A. Annaswamy, "An adaptive smith-controller for time delay systems with relative degree $n^{*} \leq 2$," Systems and Control Letters, vol. 49, pp. 347-358, 2003.

[28] X. M. Yang, X. Q. Yang, and K. L. Teo, "A matrix trace inequality," Journal of Mathematical Analysis and Applications, vol. 263, no. 1, pp. 327-331, 2001.

[29] H. G. H. Zollner, D. M. Pool, H. J. Damveld, M. M. van Paassen, and M. Mulder, "The effects of controlled element break frequency on pilot dynamics during compensatory target-following," AIAA Modeling and Simulation Technologies Conference, Aug. 2010.

[30] E. Lavretsky, and T. E. Gibson, "Projection operator in adaptive systems," arXiv ePrints, arXiv:1112.4232, 2011. 